\title{
Abondance et diversité des communautés lombriciennes dans la Réserve et Domaine de Chasse de Bombo-Lumene (Kinshasa)
}

\author{
Fils MILAU EMPWAL ${ }^{1 *}$, Aboulkacem LEMTIRI ${ }^{2}$, Carmel KIFUKIETO MANZANZA ${ }^{1}$, \\ Claude KACHAKA SUDI ${ }^{1}$, Jules ALONI KOMANDA ${ }^{3}$, \\ Erick BUKAKA WAKINI YETO ${ }^{4}$ et Fréderic FRANCIS ${ }^{2}$
}

\author{
${ }^{1}$ Laboratoire de Gestion des Ressources Naturelles, Faculté des Sciences Agronomiques de l'université de \\ Kinshasa, RD Congo. \\ ${ }^{2}$ Unité d'Entomologie Fonctionnelle et Evolutive, Gembloux Agro-Bio Tech - Université de Liège, B-5030 \\ Gembloux, Belgique. \\ ${ }^{3}$ Laboratoire de Géomorphologie et pédologie, Faculté des Sciences, université de Kinshasa, RD Congo. \\ ${ }^{4}$ Laboratoire de Bio-statistique, Faculté des Sciences, université de Kinshasa, RD Congo. \\ *Auteur correspondant, E-mail : filsmilau@gmail.com, Tél : +243 998 426 414, +243 89510249.
}

\begin{abstract}
RESUME
Les vers de terre sont des espèces importantes des écosystèmes édaphiques dotées des capacités de transformer leur biotope et même parfois de créer de nouveaux habitats pour d'autres organismes. En effet, ils modifient la morphologie, la structure et la composition du sol par leur action à la fois physique, chimique et biologique. Ils ont ainsi une implication intéressante dans la fertilité et le cycle des nutriments du sol. Cette étude a été effectuée dans la Réserve et Domaine de Chasse de Bombo-Lumene (RDCBL) à Kinshasa. Elle avait pour objectif d'évaluer l'effet de l'occupation du sol sur la composition et la structure des communautés lombriciennes. Dix-huit parcelles regroupées en 6 biotopes ont été échantillonnées: les forêts denses semi décidues, les galeries forestières, les recrûs forestiers, les savanes arborescentes, les savanes herbeuses, et les sols sous culture. Au total, 10 espèces de vers de terre ont été identifiées. Elles appartiennent aux deux grandes familles tropicales : celle des Acanthodrilidae et celle des Eudrilidae. Les biotopes naturels sont les plus riches en termes de richesse d'espèces comparés aux biotopes les plus anthropisés. Dans cette étude, le type d'occupation du sol affecte considérablement la diversité des vers de terre.
\end{abstract}

(C) 2017 International Formulae Group. All rights reserved.

Mots clés : Communautés lombriciennes, densité, richesse spécifique, Bombo-Lumene.

\section{Abundance and diversity of the lombrician communities in the Reserve and Hunting Area of Bombo-lumene (Kinshasa)}

\begin{abstract}
The earthworms are significant species of the edaphic ecosystems equipped with the capacities to transform their biotope and even sometimes to create new habitats for other organizations. Indeed, they modify morphology, the structure and the composition of the soil by their physical, chemical and biological action. They have an interesting implication in the fertility and the cycle of the nutriments of the soil. This study was carried out in the Reserve and Hunting Area of Bombo-Lumene (RDCBL) in Kinshasa. It was intended to
\end{abstract}


show the effect of land use on the diversity of earthworms. Eighteen plots grouped into six kinds of habitats were sampled (semi deciduous dense forest, gallery forest, forest regrowth, tree savannah, grassland, soil under cultivation). In total, ten earthworm species were identified. They all belong to Acanthodrilidae and Eudrilidae families. Natural habitats were the most species-rich and most diverse areas compared to the manmade habitats. The land use showed a very significant effect on the diversity of earthworm species.

(C) 2017 International Formulae Group. All rights reserved.

Keywords: Earthworms, density, species diversity, Bombo-Lumene.

\section{INTRODUCTION}

Les vers de terre occupent une place importante dans les écosystèmes. Ils jouent un rôle primordial dans l'incorporation, la transformation de ces matières et améliorent la structure du sol (Decaens et Jiminez, 2002). Par leurs activités, les lombrics sont de véritables acteurs et bioindicateurs de la fertilité des sols Miau et al. (2015). Les espèces de vers de terre retrouvées dans un milieu dépendent de la nature, des caractéristiques du sol et des conditions climatiques (Gastine, 2003). Dans les écosystèmes naturels, les communautés de vers de terre peuvent être composées d'une dizaine à une centaine d'individus par $\mathrm{m}^{2}$ selon le type de biotope (Curry, 2004). Ainsi, la distribution des espèces, leur abondance, leur biomasse, leur richesse, et leur activité en milieu naturel sont conditionnées par des facteurs biotiques et abiotiques (Kate et al., 2016).

Les vers de terre sont affectés en termes d'abondance, de biomasse et de diversité spécifique par certains facteurs environnementaux (Mamadou et al., 2014). Cependant, dans les différents biotopes tropicaux, peu d'études ont été effectués pour comprendre les mécanismes pouvant affecter les paramètres de vers de terre. En République Démocratique du Congo (RDC), Dix espèces des vers de terre ont été identifiées (Milau, 2016). Néanmoins la présence et la diversité naturelles des vers de terre dans les sols de la $\mathrm{RDC}$ restent très peu investiguées.

L'objectif du présent travail est de déterminer la diversité des espèces en fonction des différentes occupations du sol de la
Reserve et Domaine de chasse de BomboLumene.

\section{MATERIEL ET METHODES Milieu d'étude}

L'étude a été réalisée dans la Réserve et le Domaine de Chasse de Bombo-Lumene, compris entre $4^{\circ} 20^{\prime}$ et $5^{\circ} 80^{\prime}$ de latitude Sud et $15^{\circ} 50^{\prime}$ et $16^{\circ} 20^{\prime}$ de longitude Est et à $600 \mathrm{~m}$ d'altitude au plateau des Batékés. Elle est située sur la route asphaltée $n^{\circ} 1$ KinshasaKikwit, à $125 \mathrm{~km}$ du centre-ville de Kinshasa dans la commune de Maluku.

Les sols sont classifiés comme Rubique Ferralique Arénosol (Dystrique) selon le système de classification WRB (IUSS Working Group WRB, 2006), et comme Quartzipsamment Ustoxique Isohyperthermique suivant la classification américaine (Soil Survey Staff, 2006). Les sols de la couche arable ont une composition granulométrique moyenne de l'ordre de 3,4\% argile, $5,6 \%$ limon et $91,0 \%$ sable, et possèdent une densité apparente d'environ 1,25 (Kasongo et al., 2009). Le climat de la région est tropical humide du type $\mathrm{AW}_{4}$ selon la classification de Köppen c'est-à-dire avec une saison sèche de quatre mois sans pluies (Juin à Septembre), une saison pluvieuse (Octobre à avril); Mai et Octobre constituant des mois de transition (Milau et al., 2015).

$\mathrm{La}$ végétation de la Réserve et Domaine de chasse de Bombo-Lumene est diversifiée (Habiyaremye, 2011). Les biotopes étudiés sont :

- les forêts denses semi décidues: habitat le plus évolué de la série des forêts de terre ferme. Elles couvrent des superficies moins étendues que celles des savanes; 
- les galeries forestières: sont établies le long des cours d'eau et occupent les fonds des plaines de la Bombo et de la Lumene. Elles s'étendent sur une dizaine de mètres de part et d'autre des rives de ces rivières;

- les recrûs forestiers: se caractérisent par trois strates dominées par des arbres pionniers à croissance rapide tels que Dracaena mannii Dewild., Pentaclethra macrophylla Penth., Albizia adiantifolia Schumach. ,...

- les savanes arborescentes: comportent un tapis de graminées parsemées d'arbustes de moins de $7 \mathrm{~m}$ de hauteur recouvrant plus ou moins $20 \%$ de la superficie de cet habitat;

- les savanes herbeuses: sont prédominées par une couverture herbacée. Les arbres et les arbustes son absents. Elles couvrent $40 \%$ de la superficie de cette aire protégée;

- les sols sous culture: où prédominent les cultures de manioc (Manihot esculanta Crantz) et des arachides (Arachis hypogea L).

\section{Méthode d'échantillonnage et d'analyse des données}

L'extraction des lombriciens a été réalisée selon la méthode préconisée par Cluzeau et al. (1999) qui comporte deux étapes: une extraction chimique suivie d'un tri manuel sur un bloc de sol. Cette méthode repose sur l'efficacité de l'extracteur chimique. Cette dernière a été réalisée sur une surface d'un mètre carré après un triple épandage de dix litres d'une solution de formaldehyde à $4 \%$. Cette méthode est bien adaptée aux milieux hétérogènes où la densité des lombriciens est supposée être différente et la taille de la surface du prélèvement suffisante pour intégrer à la fois la variabilité de distribution des lombriciens associée à cette hétérogénéité naturelle du milieu et leur faible densité. Elle a été complétée par un tri manuel appliqué sur un volume de $0,02 \mathrm{~m}^{3}$ de sol $\left(0,10 \mathrm{~m}^{2} \times 0,2 \mathrm{~m}\right.$ de profondeur). Trois répétitions ont été réalisées sur chaque type de biotope étudié. Au total, 18 parcelles ont été investiguées. Les lombriciens récoltés étaient placés dans des piluliers préalablement étiquetés contenant une solution de formol à $4 \%$. Leur identification a été basée sur la clé de Csuzdi (2010).

Trois indices écologiques ont été utilisés pour décrire les communautés: (1) la richesse spécifique (S) qui correspond au nombre total d'espèces échantillonnées par milieu, (2) l'indice de Shannon-Weaver (H') qui prend en compte le nombre de taxons rencontrés sur la parcelle $\left(\mathrm{H}^{\prime}=-\Sigma\right.$ pi $\mathrm{x} \log 2$ (pi), pi $=$ nombre d'individus d'un taxon $\mathrm{i}$ rencontré sur une parcelle sur le nombre total d'individus pour les taxons rencontrés sur la parcelle et (3) l'équitabilité (E) correspondant à la répartition équitable des taxons permettant de comparer des peuplements comportant des nombres de taxons différents. Elle est égale au rapport entre la diversité réelle calculée et la diversité théorique maximum. Avec : $\mathrm{E}=\mathrm{H}^{\prime} / \log 2(\mathrm{~s})$, s étant le nombre de taxa observés. E tend vers 0 lorsqu'un taxon domine largement un peuplement et est égale à 1 lorsque tous les taxons ont la même abondance. Tous ces indices ont été calculés avec le logiciel Past (Hammer, 2001).

\section{Analyses statistiques}

L'effet de l'occupation du sol sur les biomasses a été testé par une analyse des variances (ANOVA) à un facteur. Si l'ANOVA indiquait un effet significatif un test de Fisher LSD a été réalisé pour comparer les moyennes des échantillons.

Une Analyse Factorielle des Correspondances (AFC) a été réalisée pour établir le lien entre les espèces lombriciennes et les biotopes qui les inféodent. Elle a été utilisée à partir des données d'abondances relatives des espèces de vers de terre. Toutes ces analyses ont été réalisées avec le logiciel Xlstat 2014. Le logiciel Origin (version 6.1) a été utilisé pour les présentations graphiques.

\section{RESULTATS}

Abondance des espèces lombriciennes Des différences significatives d'abondances des communautés 
lombriciennes ont été observées entre les biotopes étudiés $(\mathrm{p}<0,001)$ (Figure 1). La forêt dense semi décidue abrite les abondances les plus importantes de vers comparée aux autres biotopes. Ces abondances n'ont pas révélé de différences significatives d'une part entre la galerie forestière et la savane arborescente, entre le recrû forestier et la savane herbeuse et entre la savane herbeuse et le sol sous culture d'autre part.

\section{Richesse spécifique}

Un total de 10 espèces de vers de terre a été recensé dans l'ensemble des biotopes (Tableau 1). Elles appartiennent à 2 familles: Acanthodrilidae (Dichogaster wenkei, D. tenuiseta, $D$. navana, $D$. savanicola, $D$. silvestris, D. stuhlmanni, D. austeni, $D$. congica et Benhamia itoliensis) et Eudrilidae (Hyperiodrilus africanus). Ces espèces sont en majorité endémiques de la RDC exceptées Hyperiodrilus africanus et Benhamia itoliensis. La comparaison de cette richesse spécifique entre tous les biotopes présente de grandes variabilités. Sept espèces ont été rencontrées dans la galerie forestière et 6 dans la forêt dense semi décidue. Le recrû forestier est associé à une richesse de 5 espèces. Trois espèces ont été identifiées concomitamment dans la savane arborescente et herbeuse; 2 espèces ont été identifiées dans le sol sous culture. En fonction du niveau d'intégration considéré, les différences apparaissent au niveau de chaque biotope. Sur les 10 espèces recensées, 7 ont été considérées comme espèces sensibles notamment: Hyperiodrilus africanus, Benhamia itoliensis, Dichogaster wenkei, $D$. navana, $D$. silvestris, $D$. stuhlmanni, et $D$. silvesrtis. Les espèces considérées comme ubiquistes sont: $D$. congica et $D$. savanicola. Elles ont été retrouvées dans la plupart des biotopes. Cependant, l'espèce $D$. tenuiseta semble principalement inféodée à la forêt dense semi décidue. La similarité taxonomique liée aux conditions pédo-climatiques de la zone d'étude et à l'intensité de l'anthropisation est importante entre la savane herbeuse et le sol sous culture (0.67) et entre la forêt dense semi décidue et la galerie forestière (0.63). Cette similarité est moins importante entre les autres biotopes.

\section{Diversité spécifique et équitabilité}

L'indice de diversité de Shannon suit la même évolution que la richesse spécifique (Figure 2). Les valeurs les plus élevées ont été observées dans la galerie forestière et la forêt dense semi décidue avec, respectivement $\mathrm{H}^{\prime}=$ 2.78 et $\mathrm{H}^{\prime}=2.47$; la plus faible valeur avait été observée dans le sol sous culture $\left(H^{\prime}=1\right)$. L'équitabilité varie faiblement, la valeur la plus élevée a été observée dans le sol sous culture $(E=1)$, suivie par la galerie forestière $(\mathrm{E}=0.99)$. La savane arborescente et la savane herbeuse ont respectivement des valeurs de $E$ égales à 0.91 et à 0.83 et paraissent comme les biotopes les moins équilibrés.

\section{Corrélation entre les biotopes et les espèces lombriciennes}

Une AFC basée sur l'abondance relative de chaque espèce dans les différents biotopes apporte une réponse plus précise aux observations faites sur la distribution des espèces au sein des biotopes (Figure 3). Suivant l'analyse des valeurs propres, les deux premiers axes expliquent $74.54 \%$ de la variance totale de l'échantillon. L'axe 1 présente deux groupes nettement distincts. Le premier est composé de $D$. silvestris, $D$. tenuiseta et $D$. wenkei, significativement corrélées avec la forêt dense; le deuxième groupe isole $D$. savanicola, associée à la savane arborescente. L'axe 2 révèle une corrélation significative, d'une part, entre $H$. africanus et le recrû forestier, et d'autre part, entre $D$. congica et la savane herbeuse et le sol sous culture. 


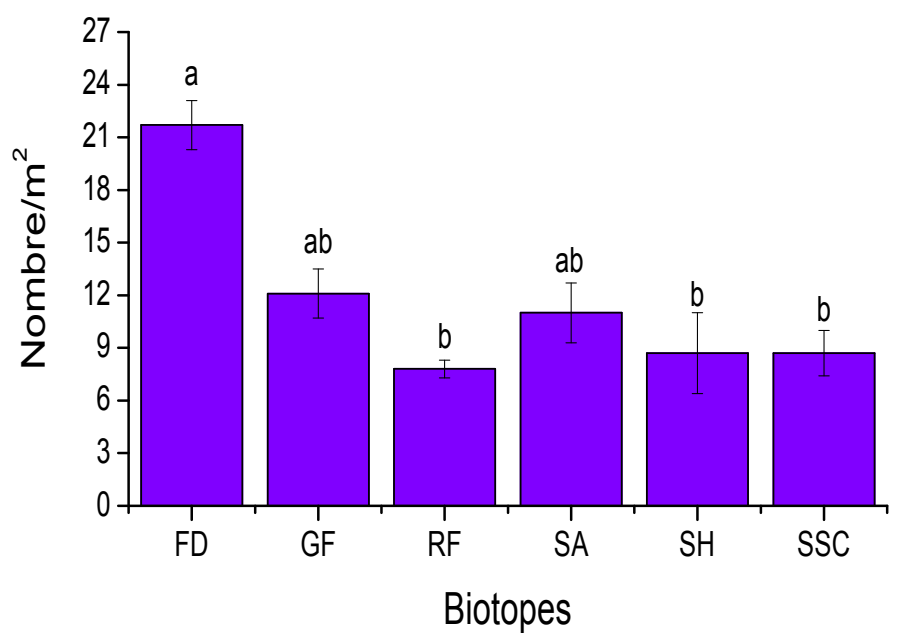

Figure 1 : Densité moyenne des vers de terre par biotope. (FD= Forêt dense semi-décidue, GF= Galerie forestière, $\mathrm{RF}=$ Recrû forestier, $\mathrm{SA}=$ Savane arborescente, $\mathrm{SH}=$ Savane herbeuse, $\mathrm{SSC}=\mathrm{Sol}$ sous culture). Les moyennes représentées par une même lettre ne se différencient pas significativement au seuil de probabilité de $5 \%$.

Tableau 1 : Diversité et abondance des taxons de vers de terre par biotopes.

\begin{tabular}{lllllll}
\hline Espèces & FD & GF & RF & SA & SH & CU \\
\hline Hyperiodrilus africanus & 0 & 0 & 29 & 17 & 0 & 0 \\
Benhamia itoliensis & 0 & 46 & 0 & 0 & 10 & 0 \\
Dichogaster Wenkei & 74 & 52 & 0 & 0 & 0 & 0 \\
Dichogaster tenuiseta & 46 & 0 & 0 & 0 & 0 & 0 \\
Dichogaster navana & 0 & 55 & 32 & 0 & 0 & 0 \\
Dichogaster savanicola & 0 & 0 & 12 & 54 & 37 & 21 \\
Dichogaster silvestris & 94 & 49 & 0 & 0 & 0 & 0 \\
Dichogaster stuhlmanni & 44 & 36 & 38 & 0 & 0 & 0 \\
Dichogaster austeni & 47 & 48 & 39 & 0 & 0 & 0 \\
Dichogaster congica & 80 & 45 & 0 & 38 & 29 & 27 \\
\hline Nombre total d'espèces & $\mathbf{6}$ & $\mathbf{7}$ & $\mathbf{5}$ & $\mathbf{3}$ & $\mathbf{3}$ & $\mathbf{2}$ \\
\hline
\end{tabular}

$\mathrm{FD}=$ Forêt dense semi-décidue, $\mathrm{GF}=$ Galerie forestière, $\mathrm{RF}=$ Recrû forestier, $\mathrm{SA}=$ Savane arborescente, $\mathrm{SH}=\mathrm{Savane}$ herbeuse, $\mathrm{SSC}=$ Sol sous culture. 


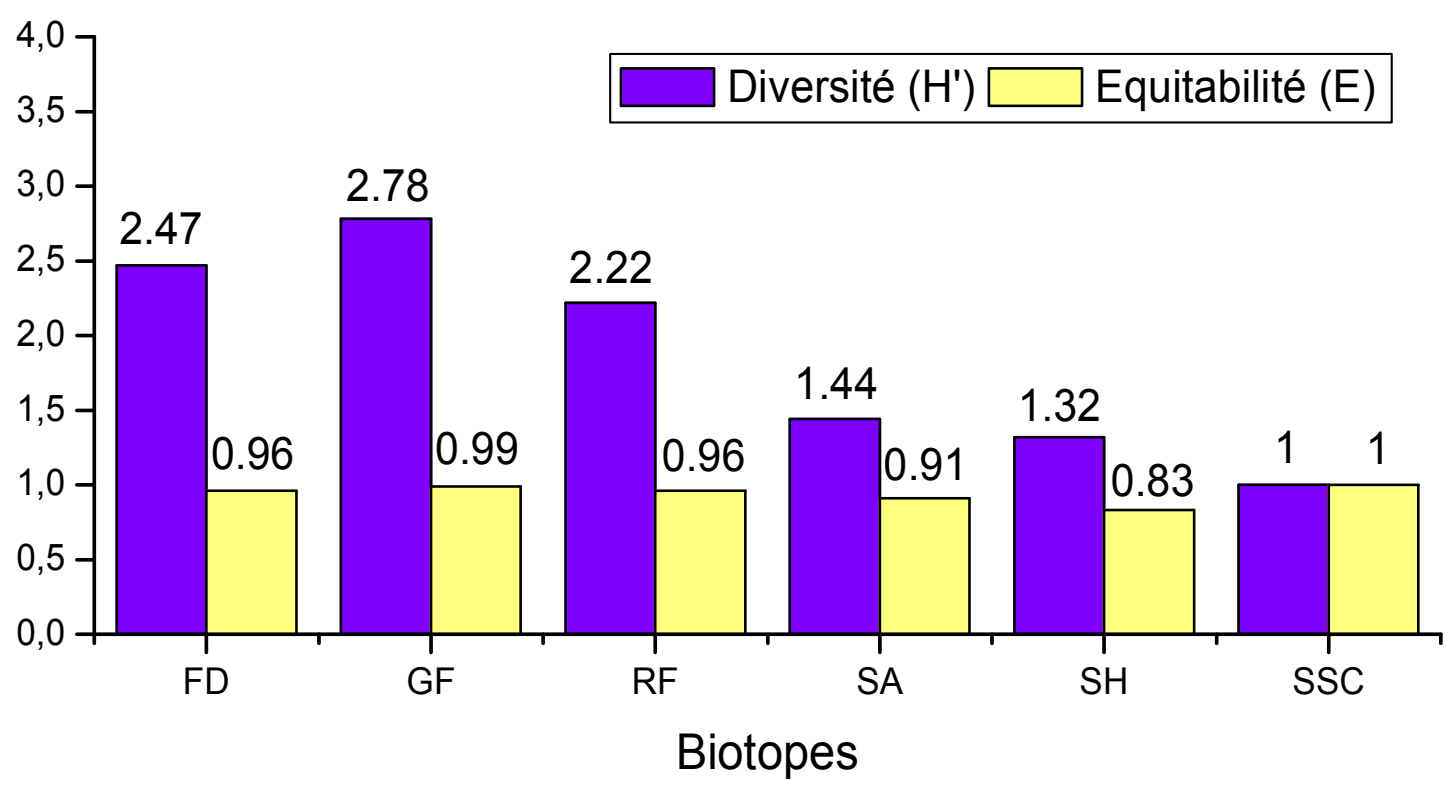

Figure 2. Indices de diversité de Shannon et d'équitabilité de Piélou des biotopes. (FD= Forêt dense semi-décidue, $\mathrm{GF}=$ Galerie forestière, $\mathrm{RF}=$ Recrû forestier, $\mathrm{SA}=$ Savane arborescente, $\mathrm{SH}=$ Savane herbeuse, $\mathrm{SSC}=\mathrm{Sol}$ sous culture).

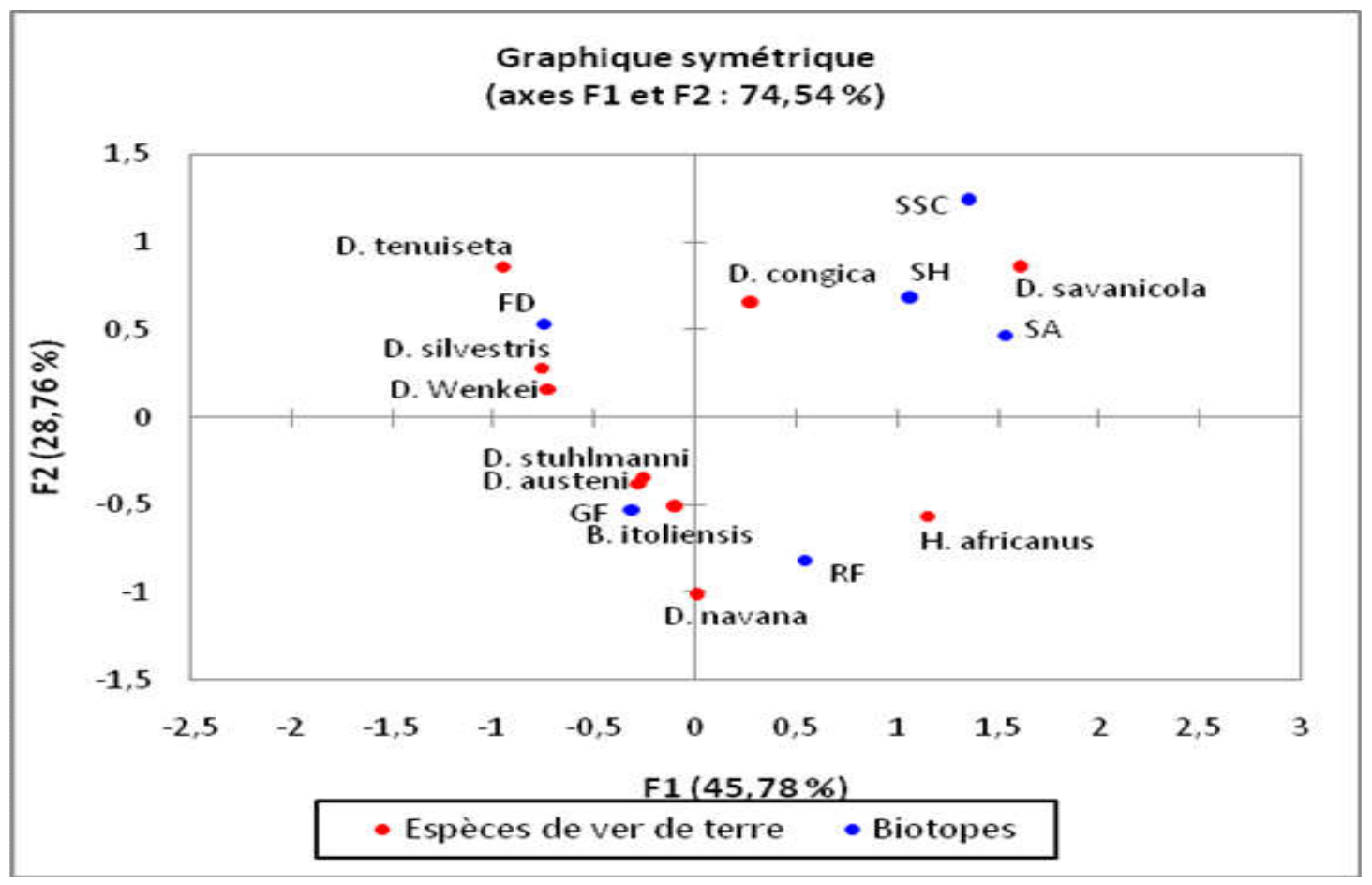

Figure 3. Illustration de l'Analyse Factorielle des Correspondances (AFC) en fonction des biotopes et des espèces de ver de terre. ( $\mathrm{FD}=$ Forêt dense semi-décidue, $\mathrm{GF}=\mathrm{Galerie}$ forestière, $\mathrm{RF}=$ Recrû forestier, $\mathrm{SA}=$ Savane arborescente, $\mathrm{SH}=$ Savane herbeuse, $\mathrm{SSC}=\mathrm{Sol}$ sous culture). 


\section{DISCUSSION}

La différence d'abondance lombricienne dans les biotopes est significative. La forêt dense semi décidue a abrité une abondance significativement plus importante contrairement à la savane herbeuse, au sol sous culture et au recrû forestier. Les valeurs d'abondances intermédiaires ont été observées dans la galerie forestière et à la savane arborescente. $\mathrm{La}$ forêt dense semi décidue peut être considérée comme le biotope le plus favorable aux communautés lombriciennes, suivi de la galerie forestière et de la savane arborescente. En effet, la forêt dense semi décidue ainsi que la galerie forestière subissent de faibles fluctuations des conditions climatiques grâce à leur canopées qui réfléchissent les radiations solaires, l'humidité du sol y reste ainsi très élevée. Ces facteurs peuvent favoriser la colonisation et le bon développement des communautés lombriciennes. Selon Decaëns (2010), la biodiversité des invertébrés du sol dépendent fortement de la température et de l'humidité $\mathrm{du}$ sol sont des facteurs déterminants de l'abondance et de l'activité des vers de terre et les populations lombriciennes répondent relativement rapidement à des variations de ces facteurs du milieu. Les faibles densités lombriciennes observées dans les biotopes anthropisés s'expliquent par l'intensité des pratiques agricoles qui provoquent une perturbation physique de l'habitat et une mortalité importante des vers de terre. Le travail du sol peut en effet engendrer une mortalité importante en occasionnant des blessures ou en déstructurant l'habitat physique des ces communautés (changement brutal des conditions microclimatiques, destruction de l'habitat, exposition à la prédation) (Mamadou et al., 2014 ; Noupé et al., 2014). Cette même pratique peut également diminuer la capacité de charge du système en occasionnant une diminution de la ressource trophique (taux de matière organique) ou en dégradant à plus long terme des conditions physiques du sol (Lavelle, 2001). Ces résultats sont similaires à ceux de Decaens et al. (2008) qui met en évidence que le type d'utilisation du sol a un impact significatif sur la richesse et l'abondance des espèces de vers de terre.

Concernant la richesse spécifique, les résultats obtenus dans cette étude concordent avec ceux présentés dans d'autres travaux en Afrique avec toutefois des différences ponctuelles liées à la variabilité des situations dans les études publiées sur ce sujet (Ouahrani et Gheribi, 2007). A l'échelle de la RDC, la Réserve et le Domaine de chasse de BomboLumene ne représente qu'un petit espace de 300.000 ha dans lequel 10 espèces ont été trouvées. Baha et Berra (2001) et Ouahrani (2003) ont recensé une diversité similaire avec onze espèces respectivement dans le Constantinois algérien et dans la zone de la Mitidja, une plaine côtière au Sud d'Alger.

S'agissant de la diversité et de l'équitabilité, la galerie forestière et la forêt dense semi décidue correspondent également des valeurs relativement plus élevées. Ceci peut s'expliquer par la stabilité de ces biotopes. Ce résultat est en accord avec ceux d'Eggleton et al. (2002) et Cheikh Amet et al. (2016) qui indiquent que les biotopes les plus stables sont remarquablement plus diversifiés en macrofaune que les biotopes perturbés par les activités anthropiques. D'après Decaens et Jiminez (2002), la diversité des vers de terre diminue donc fortement avec l'intensification des activités agricoles. Ceci implique par conséquent une dépendance des communautés lombriciennes vis-à-vis de la disponibilité des ressources alimentaires et révèle la prise en compte du gradient de végétation. A cet effet, il est bien connu que la disponibilité et la nature des ressources organiques, elles-mêmes étroitement liée aux activités anthropiques, 
influencent à la fois l'abondance et la diversité des communautés de vers de terre (Schmidt et al., 2003). C'est ce qui expliquerait le cas échéant la faible diversité observée dans les écosystèmes anthropisés. Selon Smeaton et al. (2003), on constate une diversité, une densité et une biomasse souvent inférieures en milieu cultivé car l'évolution des communautés lombriciennes est considérée pour sa valeur indicatrice de l'usage des sols. Toutefois, tous les paramètres étant hautement corrélés, il est difficile de distinguer leur contribution respective.

\section{Conclusion}

Cette étude a permis de montrer l'effet de l'occupation du sol sur la diversité des vers de terre en RDC sur les plateaux de Batéké. Les résultats obtenus indiquent que la forêt dense semi décidue, la galerie forestière et la savane arborescente abritent d'abondantes communautés lombriciennes et peuvent être considérées comme des biotopes favorables au développement des vers de terre. Concernant la richesse spécifique des lombriciens, la galerie forestière et la forêt dense semi décidue sont à la fois les biotopes les plus riches (avec respectivement 7 et 6 espèces) et les plus diversifiés. Ces biotopes constituent des aires de refuges pour les vers de terre en regroupant la majorité d'espèces rencontrées. La richesse et la diversité spécifique ont également traduit l'importance des biotopes naturels dans le développement des communautés lombriciennes et la dépendance écologique de chaque espèce.

\section{CONTRIBUTION DES AUTEURS}

LA a fait l'identification des espèces au laboratoire et a participé à la redaction du manuscrit ; KMC a participé à la campagne de collecte des données sur le terrain; KSC a assisté 'une façon ponctuelle à la révision du manuscrit et à la collecte des données. AKJ a révisé complètement le manuscrit; BWYE a fait des analyses statistiques des données et a contribué à l'interprétation des données; FF a financé la campagne de collecte des données et a participé à l'identification des spécimens au laboratoire.

\section{REMERCIEMENTS}

Nous remercions Mr Guy Mergeai de l'Université de Liège, Gembloux AgroBioTech, Unité de Phytotechnie Tropicale et Horticulture pour l'appui apporté à la réalisation de nos recherches.

\section{REFERENCES}

Baha M, Berra S. 2001. Prosellodrilus doumandjii n. sp., a new lumbricid from Algeria. Trop. Zool., 14: 87-93. DOI : http:/dx.doi.org/10.1080/03946975.2001.1 053114

Cheikh Amet, Corinne Rouland-Lefevre, Isabelle Grechi, Jean-Yves Rey, JeanFrançois Vayssières, Lamine Diame, Karamoko Diarra. 2016. Diversité, nuisances et modes de gestion des termites (Isoptera) dans les agrosystèmes sénégalais. Int. J. Biol. Chem. Sci., 10(1): 134-154.

DOI : http://dx.doi.org/10.4314/ijbcs.v0i1.10

Cluzeau D, Cannavacciulo M, Péres G. 1999. Indicateurs macrobiologiques des sols : les lombriciens - Méthode d'échantillonnage dans les agrosystèmes en zone tempérée. In $12^{\text {ème }}$ Colloque Viticole et Enologique Ed. ITV Paris, p 25-35.

Csuzdi Cs. 2010. A monograph of the Paleotropical benhamiinae earthworms (annelida: Oligochaeta, Acanthodrilidae). Budapest: Hungrian Natural History Museum, Systematic Zoology 348p.

Curry JP. 2004. Factors affecting the abundance of earthworms in soils. In Earthworm Ecology, Edwards CA (ed). CRC Press LLC. 
Decaens T, Jiminez J. 2002. Earthworm communities under an agricultural intensification gradient in Colombia. Plant and Soil, 240: 133-143. DOI : http/dx.doi.org/10.1023/A :101584461060 4

Decaëns T, Margerie P, Aubert M, Hedde M, Bureau F. 2008. Assembly rules within earthworm communities in North-Western France -A regional analysis. Appleid Soil Ecology, 39: 321-335. DOI : www.reseachgate.net /10.1016/j.apsoil.2008.01.007

Decaëns T. 2010. Macro ecological patterns in soil communities. Global Ecol. Biogeogr., 19(3): 287-302. DOI : http://onlinelibrary.wiley.com/doi/10.1111 /j.1466-8238.2009.00517

Eggleton PP, Bignell DE, Hauser S, Dibog L, Norgrove L, Madong B. 2002. Termite diversity across an anthropogenic disturbance gradient in the humid forest zone of West Africa. Agriculture, Ecosystems and Environment, 90:189202.

DOI : www.reseachgate.net/10.1016/S01678809(01)00206-7

Gastine A, Scherer-Lorenzen M, Leadley P. 2003. No consistent effects of plant diversity on root biomass, soil biota and soil abiotic conditions in temprerate grassland communties. Applied Soil Ecology, 24(3): 101-111. DOI : http//hdl.handle.net/11858/00-001M-0000000E-D04-3

Habiyaremye M, Nlandu L, Malio N. 2011. Habitats de la Réserve et Domaine de chasse de Bombo-Lumene (R.D.Congo). Lexique Kiteke des plantes observées dans ces milieux. Institut Congolais pour la Conservation de la Nature, 144p.

Hammer O, Harper, David AT, Ryan. 2001. PAST: Paleontological Statistics Software
Package for Education and Data Analysis. Palaeontologia Electronica; $368 \mathrm{P}$.

IUSS Working Group WRB. 2006. World reference base for soil resources (2nd edn). World Soil Resources Report No. 103. FAO, Rome.

Kasongo RK, Van Ranst E, Verdoodt A, Kanyankogote P, Baert G. 2009. Impact of Acacia auriculiformis on the chemical fertility of sandy soils on the Batéké plateau, D.R. Congo. Soil Use and Management, 25(2): 21-27. DOI : www. Onlinelibrary.wiley.com /10.1111/j.14752743-2008.00188.x

Kate S, Azontonde AH, Dagbenonbakin GD, Sinsin B. 2016. Effets des changements climatiques et des modes de gestion sur la fertilité des sols dans la commune de Banikoara au nord-ouest du Benin. Int. J. Biol. Chem. Sci., 10(1):154-163. DOI : http://dx.doi.org/10.4315/ijbcs.v0i1.10.

Lavelle P, Alister V. 2001. Soil Ecology. Spain. Kluwer Academic Publishers.101 Philip Drive, Norwell, MA 02061, Hardback, 684 p.

Mamadou Traore, Awa Barro, Ali Garame, Nacro Hassan Bismarck. 2014. Etude de la dynamique de la macrofaune du sol sous culture de deux variétés de patate douce (Ipomea batatas) avec utilisation des différents modes de désherbage. Int. J. Biol. Chem. Sci., 9(5): 2334-2345. DOI : http//dx.doi.org/10.4314/ijbcs.v9i5.7

Milau F, Kachaka S, Aloni K, Mvumbi M, Francis F. 2015. Incidence de la déforestation sur les catégories écologiques des vers de terre dans le domaine et Reserve de Chasse de BomboLumene (Kinshasa). Tropicultura, 33(3): 209-217.

DOI : http://hdl.handle.net/2268/186532

Milau Fils. 2016. Etude de la diversit'e des communaut'es lombriciennes dans la R'eserve et Domaine de Chasse de 
Bombo-lumene, plateau des Batékés. Biodiversité et Ecologie. Université de Kinshasa.

Noupé Diakaria, Mangomaké Koné, Tchoa Koné, Tanoh Kouakou,Yatty Kouadio. 2015. Influence des conditions pédoclimatiques sur les composantes du rendement de Jatropha curcas (L) dans les zones Nord de savane et Sud forestière de la Côte d'Ivoire. Int. J. Biol. Chem. Sci., 9(5): $\quad 1120-1129$.

DOI : http://dx.doi.org/10.4314/ijbcs.v9i5.8

Ouahrani G, Gheribi-Aoulmi Z. 2007. Apport des lombriciens à l'estimation des éléments traces $(\mathrm{Cd}, \mathrm{Cu}, \mathrm{Pb}$ et $\mathrm{Zn})$ des sols bordant l'oued Rhumel (Constantine). Ecologia Mediterranea, 33: 73-84. DOI : http://www.naturaliapublications.com/catalog/ecologiamediterranea-2007-p-30.html
Ouahrani G. 2003. Lombritechniques appliquées aux évaluations et aux solutions environnementales. Thèse de doctorat d'État. Université Mentouri, 230 p.

Schmidt O, Clements R., Donaldson G. 2003. Why do cereal-legume intercrops support large earthworm populations? Applied Soil Ecology, 22: $\quad 181-190$. DOI : 10.1016/S0929-1393(02)00131-2

Smeaton T, Daly A, Cranwell JM. 2003. Earthworm population responses to cultivation and irrigation in a South Australian soil. Pedobiol., 47: 379-385. DOI : http://reseachgate.net/10.1078/00314056-00201

Soil Survey Staff. 2006. Keys to Soil Taxonomy $\left(10^{\text {th }}\right.$ edn). Soil Conservation Service, USDA: Washington D.C. 